\title{
HRQoL and its association to clinical severity and service needs among individuals with a substance use disorder
}

\author{
ABSTRACT \\ Background: Health Related Quality of Life (HRQoL) within the field of substance abuse \\ and addiction research has received more attention the last years; however there is still a lack \\ of studying perceptions in comorbid samples, service needs within this population, and follow \\ up studies to see if these perception changes with treatment attendance. As the rate for \\ additional psychological issues and drop-out rate is known and reported high within this \\ population, these are vital areas to investigate when they enter treatment. By identifying \\ those who drop from treatment and their HRQoL perception together with their psychological \\ burden, we can also investigate if these patients receive more psychiatric program \\ recommendations by the ASAM software algorithms.
}

Method: A multi cite prospective study including a total of 175 treatment seeking individuals from three counties in mid-Norway was studied at baseline and three months after treatment initiation. Data was gathered from a structured ASAM interview and a self-report survey covering the SF-36 items regarding HRQoL. Their scores were compared to the normative Norwegian population and their own score at the three month follow-up survey.

Results: The results showed significantly lower HRQoL scores among treatment seeking individuals with a substance use disorder compared to the normative population, and high psychological burden is also associated with lower HRQoL perceptions. At follow up perceptions increased and significantly for Physical Function. In addition the low scoring HRQoL individuals also had higher drop-out rate and significantly higher on the physical items revealing the importance of assessing and meeting physical needs. In addition the individuals with the lowest HRQoL scores on the psychological items received more psychiatric program recommendation by ASAM than those with higher HRQoL perceptions.

Conclusions: The high drop out among those who scored lowest on HRQoL perceptions is of clinical importance and assessing and meeting needs to reduce drop out and provide individually tailored treatment is necessary. Both SF-36 and ASAM interview based software are considered valid and useful tools to retrieve patient centered and relevant information and should be used in a clinical setting to provide individually tailored treatment that meets their needs.

\section{Introduction}

Main outcomes from studies on patients with a substance use disorder (SUD) have traditionally focused on reduction in use, decrease in crime, and increase in productivity and social function. However there has been less attention to the patient's own perception of Health Related Quality of Life (HRQoL) within SUD research as well as in clinical practice. Even fewer take into account HRQoL research in terms of studying gender differences (Laudet et al, 2011) and comorbid samples (Lozano, Rojas \& Calderon, 2016). A study in 
south of Norway investigated QoL among inpatients with SUD and found that high psychological burden at baseline was associated with low QoL, and that females in treatment had a more positive change in QoL at follow-up (Pasareanu et al., 2015). It has been suggested that including QoL measures in measuring treatment effectiveness, and repeated measures throughout treatment would be valuable. The rate of co-occurring psychiatric disorders within drug using populations varies from $32 \%$ to $65 \%$ in national studies (Bakken et al, 2005, Stallvik \& Nordahl, 2014) and between $20 \%$ (Grant, et al., 2004) and 46\% (Schulte, et al., 2008) in international ones. The high prevalence of co-occurring psychiatric disorders suggests a vital importance to investigate the patient's own perception of their health including that of their mental health.

Interest in and assessment of QoL and HRQoL among patients with SUD and psychiatric comorbidity may help to identify specific areas that are clinically important given the high drop out among this patient group (Bakken et al, 2005; Brorson et al., 2013). Drop out research within SUD populations has previously primarily been directed towards general patient characteristics whereas more recent research indicates that other factors such as QoL and relational issues would be more valuable factors to address. Drop-out may in fact indicate a low QoL and therefore to focus on it and provide services thereafter could produce better treatment outcomes. The goal with health care is to restore individuals' healthy functioning. By measuring HRQoL we can get valid indicators of needs that can be translated into service needs and interventions. Self-assessed health statuses have also proved to be a more powerful predictor of mortality and morbidity than many other objective measures of health (DeSalvo et al, 2006). By measuring patients own perception of HRQoL we can therefore study the burden of having a SUD, how HRQoL is differentiated by gender and changes with treatment, to create optimal services to meet the patients' needs. To redirect focus from substance use centered and patient characteristics practice to a focus more towards QoL and optimal 
services provision, may contribute to reductions in drop-out from treatment. QoL have also been found to improve gradually with increased length of abstinence (Laudet et al, 2006), so to investigate patients progression and treatment outcomes repeatedly and over time is important.

This study is part of the larger multi-site validation study performed at 10 different centers in Norway in 2014(Stallvik, Gastfriend \& Nordahl, 2014), were the level of care recommendation of ASAM PPC- 2R (American Society of Addiction Medicine Patient Placement Criteria, second revised version; Mee-Lee et al., 2001) was tested. ASAM is software using a set of consensus criteria to assess patients' needs for services within substance use disorder treatment and for their co-occurring somatic and psychiatric disorders. As an extension to that study we wanted to investigate whether there were clinical meaningful differences in observed HRQoL and its relation to recommendation for psychiatric service programs. Since HRQoL scores have been found to be low within SUD populations and lower among comorbid individuals we should expect to see more psychiatric program recommendations among those with low HRQoL scores in our study if the ASAM is valid and able to discriminate between the patients’ needs.

Specific aims of this study were:

1. Describe HRQoL status and clinical characteristics at treatment inclusion

2. Observe HRQoL changes from treatment initiation to three months after

3. Identify whether those who drop out of treatment have lower perceived HRQoL than those who stay

4. Investigate if low HRQoL is associated with having a higher psychiatric burden before entering treatment and more psychiatric service recommendations by ASAM 


\section{Method}

\section{Participants}

A total of 261 treatment seeking individuals were given a HQOL survey after they completed a session with the American Society of Addiction Medicine, Patient Placement Criteria revised software interview (ASAM PPC- 2R, Mee-Lee et al., 2001). They were given verbal information about the content of the interview and survey together with written information and provided a signed consent before study inclusion. Patients agreed to accept Treatment as Usual (TAU) and recommended treatment placement, and were diagnosed with SUD or abuse according to the International Classification Diagnostic, version 10 (ICD-10: WHO, 1993) by the assessment centres who recommended treatment. The survey was left in an envelope and returned to the reception at the treatment care they attended. A total of 175 (67\%) returned their surveys at intake, and 62 (34\%) at 3-month follow-up. The study has been approved by The National Committee for Medical and Health Research Ethics (NEM).

\section{Instruments}

\section{ASAM PPC-2R}

Demographic data, including age, gender, education, work status, and marital status, were obtained along with variables of interest in regard to history of drug abuse and psychiatric condition with the use of the assessment and placement tool ASAM PPC-2R. The software interview includes previously validated tools including (in the Norwegian version) the European version of the Addiction Severity Index (EUROP-ASI; Hodgins \& El-Guebaly, 1992; Leonhard et al., 2000; Lauritzen \& Ravndal, 2004), the Clinical Institute Withdrawal Assessment for Alcohol (CIWA-AR) (Sullivan et al, 1989), and the The Clinical Institute Narcotic Assessment (CINA) Scale for Withdrawal Symptoms (Peachey \& Lei, 1988; MeeLee et al., 2001). ASAM has shown high inter rater reliability (Baker \& Gastfriend, 2003), 
good face validity (CSAT, 1995), convergent validity (Staines et al., 2003; Turner, et al., 1999), and predictive validity (Stallvik, Gastfriend \&Nordahl, 2014). In addition to recommending main level of care like outpatient or inpatients services, ASAM also recommends additional psychiatric services as required. These latter services are recommended on the basis of the patients reported symptoms and needs.

\section{HRQOL}

The 36-Item Short-Form Health Survey (SF-36) is a well used instrument to measure HRQoL and its psychometric properties has been validated (Ware \& Gandek, 1998, Loge et al. 1998). The instrument is a generic self administrative survey combined of 36 items measuring physical and mental health, which is divided into 8 subscales (Ware et al., 1998; Ware et al., 2000). The first four subscales are Physical function (PF), Physical Role (PR), Bodily Pain (BP), and General Health (GH). These four make up the Physical Component Summary (PCS) since they correlate most with physical component. The other 4 subscales create the Mental Component Summary Scale (MCS) which include the subscale Vitality (V), Social Function (SF), Mental Health (MH), and Role Emotional (RE). The items are answered with a “yes” or "no" on a three or six point scale. That left us with 57 respondents at follow-up, a reduction of 5 patients, due to missing data. Each subscale was recoded into a scale from 0 100, and 0 represent worst possible perceived health. Scale scores represent average for all items in the scale that the respondents answer and give the 8-scale profile of physical and mental health as a psychometric-based score. It was constructed to satisfy minimum psychometric standards necessary for group comparisons and the 8 subscales included were the ones most frequently measured and those most affected by disease and treatment (Ware et al., 1993). HRQoL was recoded into low (0-30), moderate (31-50) and high (51-100) score to investigate group differences in our study. When measuring HRQoL status among clinical populations, data from a normative general population can make clinical interpretations and 
comparisons more meaningful. In Norwegian such a reference population exists, Loge and Kaasa (1998) including 3500 Norwegians aged 19-80 which we compared our study sample against.

\section{Statistical analysis}

Mean distribution and percentages are used to present demographic and clinical variables. Ttests are used to investigate differences between continuous variables and Chi-square on categorical variables. Paired sample t-test was used to explore change in HRQoL from treatment initiation to follow-up three months after.

\section{Results}

\section{Study sample}

Out of the 175 included 115 (65.7\%) were men and 60 were women. Mean age was 32.5 with no gender difference. Single respondents $77.1 \%$ constituted the majority, $10.3 \%$ were married, and $12.6 \%$ separated/divorced. Mean duration of education was 10.8 years ( $\mathrm{SD}=1.903)$. Unemployment was reported by $44 \%, 19 \%$ were retired or on disability pension and $23 \%$ worked full time. Of the total sample 36\% lived alone and 10.9\% had no stable living arrangement, $32 \%$ were living with their partners with/without children, and $14.2 \%$ were living with other family members or friends. From outpatient services we had 55 patients and 120 (68.6\%) were inpatient responders. A mixed patient group regarding substance use is represented in this study with; $30.3 \%$ having alcohol as main drug of choice, $18.9 \%$ have opiates; stimulant is reported by $34.9 \%$, sedative by $6.9 \%$, and finally cannabis by $6.9 \%$.

\section{HRQoL status at baseline}

The respective means for the scales in the Physical Component scale and Mental Component scale is shown in table 1 , and the results are compared with the normative population in Norwegian national sample study (Loge \& Kaasa, 1998). We can see that the SUD population scores significantly lower on all SF-36 items compared to the normative population scales 
(Table 1). There were significant gender differences on four of the SF-36 items. Significant differences were seen for the variable Physical Function, Role Emotional, Bodily Pain, and Mental Health, and Mental Component Summary.

\section{Medical issues}

Over half $(51.7 \%, \mathrm{n}=175)$ were worrying about their medical problems which can influence how they perceive their physical health and HRQoL and $54 \%$ thought it was important to get treatment for their medical problems. Females found it more important than men to get medical help for their physical problems ( $\left.x^{2}=11.388(4), \mathrm{p}=.023\right)$.

\section{Psychological issues}

Over 74.3\% ( $\mathrm{n}=175)$ reported that they had been troubled by psychological problems overall, and $86.9 \%$ said it was important to get counselling. On the question; "Do you have a psychiatric diagnosis” 60\% (105) reported yes (61 males, 44 females) and more females confirmed this $\left(x^{2}=7.914(1), \mathrm{p}=.005\right)$. When asked if they were receiving the psychiatric services they needed ( $n=172) 46.9 \%$ said no, and males reported significantly more than females that they were not getting the services they needed $\left(x^{2}=11.008(2), \mathrm{p}=.004\right)$. 
Table 1 SUD population versus normative data and gender differences on SF-36 at baseline

\begin{tabular}{|c|c|c|c|c|c|c|c|c|c|c|}
\hline & $\mathbf{N}$ & MIN & MAX & $M$ & SD & $\begin{array}{l}\text { t-test gender differences } \\
t(d f), p, d\end{array}$ & Paired t-test $\mathrm{f} / \mathrm{u}$ & & \multicolumn{2}{|c|}{$\begin{array}{l}\text { Normative data(Loge \& Kaasa, } \\
\text { 1998) Normative data vs sample } \\
\text { t(df),p }\end{array}$} \\
\hline SF-36 (0-100) & & & & & & & $\begin{array}{l}\text { Men } \\
37\end{array}$ & $\begin{array}{l}\text { Women } \\
17\end{array}$ & $\begin{array}{l}\text { Men } \\
M(S D)\end{array}$ & $\begin{array}{l}\text { Women } \\
M(S D)\end{array}$ \\
\hline $\begin{array}{l}\text { Physical } \\
\text { Function PF }\end{array}$ & 169 & 0.00 & 100 & 81.80 & 18.776 & $-2.551(98.146), .012,0.40$ & NS & NS & $\begin{array}{l}89.8(15.5) \\
3.083(128)^{*}\end{array}$ & $\begin{array}{l}84.8(20.8) \\
2.945(63)^{*}\end{array}$ \\
\hline $\begin{array}{l}\text { Role_Physical } \\
\text { RP }\end{array}$ & 173 & 0 & 100 & 49.28 & 41.801 & NS & $-2.526(36), .016^{*}$ & NS & $\begin{array}{l}80.5(33.6) \\
7.154(129)^{* * *}\end{array}$ & $\begin{array}{l}75.4(37.7) \\
5.498(62)^{* * *}\end{array}$ \\
\hline RE & 172 & 0 & 100 & 45.93 & 42.688 & $-2.245(170), .026,0.14$ & NS & NS & $\begin{array}{l}84.5(29.7) \\
8.252(126)^{* * *}\end{array}$ & $\begin{array}{l}79.1(34.6) \\
7.728(60)^{* * *}\end{array}$ \\
\hline SF & 169 & 0 & 100 & 54.51 & 27.967 & NS & $-2.165(35), .037^{*}$ & NS & $\begin{array}{l}87.6(20.9) \\
11.991(125)^{* * *}\end{array}$ & $\begin{array}{l}83.7(23.1) \\
8.037(58)^{* * *}\end{array}$ \\
\hline BP & 174 & 0 & 90 & 60.51 & 24.762 & $-2.672(172), .008,0.42$ & NS & NS & $\begin{array}{l}77.2(25.0) \\
5.498(138)^{* * *}\end{array}$ & $\begin{array}{l}73.0(26.6) \\
5.889(66)^{* * *}\end{array}$ \\
\hline MH & 171 & 0 & 100 & 53.43 & 21.873 & $-2.542(169) .012,0.41$ & NS & NS & $\begin{array}{l}80.0(15.8) \\
10.785(12 \\
3)^{* * *}\end{array}$ & $\begin{array}{l}77.6(17.0) \\
11.761(61)^{* * *}\end{array}$ \\
\hline v & 171 & 0 & 100 & 40.09 & 21.451 & NS & NS & NS & $\begin{array}{l}63.2(19.9) \\
9.647(130)^{* * *}\end{array}$ & $\begin{array}{l}56.9(21.2) \\
8.024(64)^{* * *}\end{array}$ \\
\hline GHP & 170 & 0 & 100 & 56.53 & 22.552 & NS & NS & NS & $\begin{array}{l}77.4(21.3) \\
8.504(133)^{* * *}\end{array}$ & $\begin{array}{l}76.3(22.5) \\
7.868(62)^{* * *}\end{array}$ \\
\hline PCS & 162 & 1,25 & 98,50 & 62.69 & 21.220 & NS & NS & NS & - & - \\
\hline MCS & 163 & 0 & 97.5 & 49.18 & 23.703 & $-2,026(113,72), .045,0.32$ & NS & NS & - & \\
\hline
\end{tabular}

${ }^{*} \mathrm{P}<.01 ; * * \mathrm{p}<.001 ;{ }^{* * *} \mathrm{p}<.0001 ; \mathrm{NS}:$ Not significant 


\section{HRQoL changes from treatment initiation to follow-up}

We investigated how HRQoL changed from baseline to follow-up and the results revealed a significant improvement on the variable Physical function from baseline ( $M=83.77$, $S D=15.563)$ to follow-up ( $M=88.16, S D=14.597)$, $\mathrm{t}=-2.225(56), \mathrm{p}=.030$.

\section{HRQoL among drop-out versus retainers}

At follow-up 119 of 175 were still in treatment and 57 (32.6\%) had dropped out of treatment and/ or were actively using drugs. We investigated if there was a difference in HRQoL perceptions at baseline among those who dropped out versus those who remained in treatment. By using the recoded HRQoL items we were able to investigate if the lowest HRQoL reporting patients also drop out more. Significant differences between the three groups on the item Physical Function $\left(X^{2}=7.023(2), \mathrm{p}=.030\right)$, Role Physical $\left(X^{2}=6.079(2)\right.$, $\mathrm{p}=.048)$ and Bodily Pain $\left(X^{2}=8.471(2), \mathrm{p}=.014\right)$ emerged. Those with the low HRQoL on Physical function showed more drop out (69.4\%) compared to those with high HRQoL scores were only 30.6\% dropped out. On Role Physical 39.7\% with low HRQoL drop out versus $31.2 \%$ among those with high HRQoL. The results indicate a higher proportion of drop out among those with the lowest HRQoL scores and higher percentage of patients who remain in treatment among those with moderate to high HRQoL scores baseline scores.

\section{HRQoL and psychiatric burden and service recommendations}

Comparing the mean scores on the HRQoL items of those who report having a psychiatric diagnosis and those who report no diagnosis indicate that patients with a psychiatric diagnosis report significant lower on a majority of the HRQoL items (table 2).

In addition to psychiatric burden and its associations, we also hypothesized that low scores on the mental items in particular of HRQoL also would be associated with more psychiatric services recommendations. ASAM recommendations showed that 114 (65.1\%) received such additional service recommendations. A Chi square analysis revealed no gender differences in 
these recommendations. Patients with psychiatric services recommendations scored lower on all HRQoL items compared to those who did not receive such recommendation and significantly lower on the variables:

Role Emotional $(\mathrm{N}=113): M=41.00(S D=40.339), \mathrm{t}(105.644)=2.034, \mathrm{p}=.044, d=0.33$

Social Functioning $(\mathrm{N}=111): M=50.34(S D=28.428), \mathrm{t}(167)=2.735, \mathrm{p}=.007, d=0.44$

Mental Component Summary (N=108): $M=45.83(S D=23.238), \mathrm{t}(161)=2.573, \mathrm{p}=.011$, $d=0.42$

Among the patients with the lowest HRQoL scores on Role Emotional (0-30) there was $83.8 \%$ that received psychiatric service recommendations, compared to $68.2 \%$ in the moderate group and 57.7\% in the highest HRQoL group. On the variable Social Function a significant differences were detected $\left(X^{2}=8.511(2), \mathrm{p}=.014\right)$ and we see a greater proportion psychiatric service recommendation in the lowest HRQoL group (85\%) compared to the moderate group (60.4\%) and those with highest HRQoL scores (59.8\%). 
Table 2 Independent sample t-test psychiatric severity and HRQoL variables

\begin{tabular}{|c|c|c|c|c|c|}
\hline HRQoL & $\mathbf{N}$ & $M$ & SD & t-test(df),p & Cohens d \\
\hline \multicolumn{6}{|c|}{ Psychiatric diagnosis } \\
\hline Physical_function & 105 & 78.29 & 20.741 & $3.237(98.145), .002$ & 0.55 \\
\hline Role_physical & 107 & 44.16 & 42.557 & ns & - \\
\hline Role_Emotional & 107 & 43.30 & 42.760 & ns & - \\
\hline Social_function & 106 & 50.94 & 29.363 & $1.999(84.893), .049$ & 0.35 \\
\hline Bodily_pain & 108 & 54.70 & 26.092 & $4.055(90.253),<.0001$ & 0.70 \\
\hline Mental_health & 104 & 48.65 & 22.511 & $2.961(141), .004$ & 0.58 \\
\hline General Health & 104 & 54.42 & 23.341 & ns & - \\
\hline \multicolumn{6}{|l|}{ Perception } \\
\hline Physical Health & 100 & 58.93 & 22.687 & $2.529(74.816), .014$ & 0.47 \\
\hline Mental Health & 101 & 46.09 & 24.166 & $1.942(136), .054$ & 0.38 \\
\hline \multicolumn{6}{|c|}{ Suicide tried } \\
\hline Physical_function & 67 & 75.82 & 21.683 & $3.239(109.594), .002$ & 0.53 \\
\hline Role_physical & 70 & 38.57 & 37.628 & $2.833(171), .005$ & 0.44 \\
\hline Role_emotional & 69 & 38.16 & 40.534 & $1.997(152.757), .048$ & 0.31 \\
\hline Social_function & 69 & 46.74 & 27.922 & $3.076(167), .002$ & 0.48 \\
\hline Bodily_pain & 71 & 55.45 & 25.273 & $2.265(172), .025$ & 0.35 \\
\hline Mental_health & 67 & 45.49 & 23.239 & $3.969(169), p<.0001$ & 0.61 \\
\hline Vitality & 67 & 33.28 & 22.773 & 3.433(169),.001 & 0.53 \\
\hline General Health & 66 & 49.92 & 22.283 & $3.120(168), .002$ & 0.49 \\
\hline \multicolumn{6}{|l|}{ Perception } \\
\hline Physical Health comp. & 62 & 56.56 & 21.223 & $2.964(160), .004$ & 0.48 \\
\hline summary & & & & & \\
\hline Mental Health comp. & 63 & 41.91 & 23.268 & $3.196(161), .002$ & 0.51 \\
\hline summary & & & & & \\
\hline
\end{tabular}




\section{Discussion}

Our study shows low HRQoL and high psychological burden at baseline, among persons approaching substance use disorder services. Following treatment we see improvements on all HRQoL items, although not significant changes for all subscales. Those with the lowest HRQoL perceptions dropped out from treatment more often compared to higher HRQoL scoring individuals. High psychological burden and low HRQoL is associated with more psychiatric services recommendations by ASAM.

Our results revealed improvements in all HRQoL items at follow-up and a significant improvement in the patients' perception of Physical function. Vederhus and colleagues (2016) found that QoL improved at follow-up, but the SUD sample scores were still significantly lower than for the non-SUD medical sample, which might explain why we only see a significant improvement in one of the eight HRQoL scores. The fact that SUD is a multifaceted disease which takes time to improve implies that physical and mental health is vital to consider when providing optimal services for this group.

Persons dropping out from treatment (32.6\%) in our study had lower HRQoL scores whereas those with moderate to high HRQoL seemed to remain in treatment more often. This indicates that the needs among those with low HRQoL perceptions are not adequately met and suggests a need for better individually tailored treatment for this group to ensure treatment attendance. As a group these individuals scored consistently lower on the physical health variables which indicate that services affecting these needs are important for their treatment outcomes. The longer patients stay abstinent the higher is their chances to experience HRQoL improvement, since QoL has been found to improve gradually with increased length of abstinence (Laudet et al, 2006).

The worries about health and treatment needs became even clearer when analyzing the data for psychological issues in our study. Over $74 \%$ reported worries and $87 \%$ expressed need for counselling for their psychological issues. More males report that they were not getting the psychiatric services that they perceived needed, which might imply that their potential diagnosis were undetected. With more assessment of these needs for males among SUD patients they might increasingly get appropriate diagnosis, or at least their mental health assessed for optimal treatment planning. Although women reported higher psychiatric burden, their needs in this area seemed to be more often attended. This might be caused by their better 
ability to report their symptoms to clinical personnel, resulting in treatment services that more often fulfill their needs.

Having high psychological symptom burden when entering treatment has been associated with low QoL (Pasareanu et al., 2015) and low HRQoL (Lozano et al., 2016) and this was also the case for the patients in our study. Having a psychiatric diagnosis and additional psychological services recommendations is significantly related to lower HRQoL scores. For the lowest scoring HRQoL patients we observed more recommendations for psychiatric services, and this result lends further support for the discriminate validity of ASAM in assessing needs for psychiatric challenges among patients with a SUD. Since low HRQoL is associated with higher drop-out and more psychological service recommendations, it is therefore considered an important area to investigate further. It seems the application of both HRQoL and the ASAM tool when providing services for this group can improve knowledge and understanding of needs and thereby to provide optimized services, adapted to individual needs. Patients with ASAM recommendation for psychiatric services compared to those without had significant lower HRQoL than those without on the HRQoL mental components. This further supports ASAM criteria's ability to distinguish those in need for these types of services. Knowing the high dropout rate among comorbid patients it is of great importance that their needs are assessed thoroughly (Stallvik \& Nordahl, 2014). A low score in HRQoL and high psychological burden in addition to SUD should, as a standard in clinical SUD settings, be assessed thoroughly and met with optimal services to enhance treatment attendance and thus outcomes. Reducing bodily pain perception through aerobic exercise(Hoffman \&Hoffman, 2007) or adopting mindfulness-based interventions for chronic or acute pain might be examples of interventions to enhance treatment retention among these low scoring HRQoL patients(Salomons \& Kucyi, 2011).

A survey like SF-36 or another general QoL questionnaire should be considered in clinical settings to measure patients' perceptions when they enter and how their perceptions change during treatment. By using these smaller instruments in clinical setting it can be used to measure patients’ progression repeatedly during treatment to give them feedback and rearrange treatment according to changed needs and progression. Repeated measures with easily administered instrument measuring HRQoL are clinically useful in additions to the more comprehensive level of care instrument like ASAM. 


\section{Implication}

The application of screening tools and questionnaires such as the SF-36 to explore patients' HRQoL should be standard clinical procedures and repeated measures allows monitoring of treatment outcome development. By using the ASAM criteria and the algorithms the software is based on, one can discriminate among those with low and high severity profile and recommend the optimal intensity in level of care to provide better treatment outcomes for the patients and treatment utilization for service providers.

\section{Conclusion}

Patients entering SUD treatment have severe reductions in HRQoL domains and commonly experiencing psychiatric burden in addition to the substance use disorder. The need for increased attention towards HRQoL perceptions, somatic and psychological issues is apparent, to improve treatment outcomes. The high drop out among those who scored lowest on HRQoL perceptions is of clinical importance and assessing and meeting needs to reduce drop out and provide individually tailored treatment which can increase these perception. Both SF-36 and ASAM interview based software are considered valid and useful tools to retrieve patient centered and relevant information and should be used in a clinical setting to guide treatment to ensure that patients' needs are being met. 


\section{References:}

Baker SL \& Gastfriend DR. Reliability of multidimensional substance abuse treatment matching: Implementing the ASAM patient placement criteria. Journal of Addictive Disorders. 2003, 22(1), 45-60.

Bakken K, Landheim AS \& Vaglum P. Substance-dependent patients with and without social anxiety disorder: Occurrence and clinical differences: A study of a consecutive sample of alcohol-dependent and poly-substance-dependent patients treated in two counties in Norway. Drug and Alcohol Dependence. 2005, 80, 321-328.

Brorson HH, Arnevik EA, Rand-Hendriksen K \& Duckert F. Drop-out from addiction treatment: A systematic review of risk factors. Clinical Psychology Review,2013, 1010-1024. doi: 10.1016/j.cpr.2013.07.007

CSAT, Center for Substance Abuse Treatment. The role and current status of patient placement criteria in the treatment of substance use disorders. Treatment Improvement Protocol (TIP). 1995, Rockville: Substance Abuse Mental Health Services Administration.

DeSalvo KB, Bloser N, Reynolds K, He J, Muntner PJ. Mortality prediction with a single general self-rated health question. A meta-analysis. Gen Intern Med. 2006, 21(3):267-75.

Gonzales R, Alfonso A, Marinelli-Casey P, Deborah GC, Iguchi MY, Rawson RA. Healthrelated quality of life trajectories of methamphetamine-dependent individuals as a function of treatment completion and continued care over a 1-year period. Journal of Substance Abuse Treatment. 2009, 37(4):353-361.

Grant BF, Stinson FS, Dawson DA, Chou SP, Dufour MC, Compton W, ...Kaplan K. Prevalence and co-occurrence of substance use disorders and Independent mood and anxiety disorders: Results from the national epidemiologic survey on alcohol and related conditions. Archives of General Psychiatry. 2004, 61(8), 807816. doi: 10.1001/archpsyc.61.8.807. 
Hodgins DC \& El-Guebaly N. More data on the Addiction Severity Index. Reliability and validity with the mentally ill substance abuser. Journal of Nervous and Mental Disorders. 1992, 180(3):197-201.

Hoffman MD\& Hoffman DR. Does aerobic exercise improve pain perception and mood? A review of the evidence related to healthy and chroni pain subjects. Current Pain and Headache Reports, 2007, 11(2),93-97.

Laudet AB, Morgen K, White WL. The role of social supports, spirituality, religiousness, life meaning and affiliation with 12-step fellowships in quality of life satisfaction among individuals in recovery from alcohol and drug problems. Alcoholism Treatment Quarterly. 2006;24(1-2):33-73.

Laudet AB. The case for considering quality of life in addiction research and clinical practice. Addiction science \& clinical practice. 2011;6(1):44-55.

Lauritzen G \& Ravndal E. Introduction of the EuropASI in Norway, Clinical and research experiences from cost-effectiveness study. Journal of Substance Use. 2004, 9(3-4), 141-146.

Leonhard C, Mulvey K, Gastfriend DR, Shwartz M. The Addiction Severity Index: A field study of internal consistency and validity. Journal of Substance Abuse Treatment. 2000;18(2):129-35.

Loge JH \& Kaasa S. Short Form 36 (SF-36) health survey: normative data from the general Norwegian population. Scandinavian Journal of Public Health. 1998 ,26, 250-258.

Lozano OM, Roja AJ, Calderon FF. Psychiatric comorbidity and severity of dependence on substance users: how it impacts on their health-related quality of life? Journal of Mental Health, 2016, early online: 1-8, doi: 10.1080/09638237.2016.1177771

Mee-Lee D, Shulman GD, Fishman M, Gastfriend DR \& Griffith JH. ASAM PPC-2R. ASAM Patient Placement Criteria for the treatment of Substance-Related Disorders. American Society of Addiction Medicine, Inc. Chevy Chase,Maryland, 2001, 1-50. 
Pasareanu AR, Opsal A, Vederhus JK, Kristensen O, Clausen T. Quality of life improved following in-patient substance use disorder treatment. Health and quality of life outcomes. 2015;13:35.

Peachey J \& Lei H. Assessment of Opioid dependence with naloxone. British Journal of Addiction. 1988, 83(2), 193-201.

Salomons TV \& Kucyi A. Does mediation reduce pain through a unique neural mechanism? Journal of Neuroscience. 2011,31(36), 12705-7. Doi: 10.1523/JNEUROSCI.2843-11.2011.

Schulte S, Meier PS, Stirling J, \& Berry M. Treatment approaches for dual diagnosis clients in England. Drug and Alcohol Review. 2008,27(6), 650- 658.

Staines G, Kosanke N, Magura S, Bali P, Foote J \& Deluca A. Convergent Validity of the ASAM patient placement criteria, using a standardized computer algorithm. Journal of Addictive Disorders. 2003, 22(1), 61-77.

Stallvik M \& Nordahl HM. Convergent validity of the ASAM criteria in co-occurring Disorders. Journal of Dual Diagnosis. 2014, 10(2), 68-78.

doi:10.1080/15504263.2014.906812.

Stallvik M, Gastfriend DR \& Nordahl HM. Assessing Patients with a substance use disorder to optimal level of care with the ASAM criteria software. Journal of Substance Use, 2014, 110. doi:10.3109/14659891.2014.934305.

Sullivan JT, Sykora K, Schneiderman J, Naranjo CA, \& Sellers EM. Assessment of alcohol withdrawal: The revised Clinical Institute Withdrawal Assessment for Alcohol Scale (CIWAAR). British Journal of Addiction. 1989, 84, 1353-1357.

Turner WM, Turner KH, Reif S, Gutowski WE \& Gastfriend DR. Feasibility of multidimensional substance abuse treatment matching: automating the ASAM Patient Placement Criteria. American Society of Addiction Medicine. Drug and Alcohol Dependence. 1999, 55 (1-2):35-43. 
Vederhus JK, Birkeland B, Clausen T. Perceived quality of life, 6 months after detoxification: Is abstinence a modifying factor? Qual. Life Res. 2016.

Ware JE, Snow KK, Kosinski M, Gandek B. SF-36 Health Survey Manual and Interpretation Guide. The Health Institute, 1993, Boston, MA.

Ware JE \& Gandek B, Project I. Overview of the SF-36 Health Survey and the International Quality of Life Assessment (IQOLA) Project. J Clin Epidemiol. 1998;51:903-12. doi: 10.1016/S0895-4356(98)00081-X.

Ware JE Jr, Kosinski M, Dewey J. How to score version two of the SF-36 health survey. Lincoln, RI: QualityMetric Inc; 2000.

Wills TA \& DePaulo BM. Interpersonal analysis of the help seeking process. In CR Snyder \& DR Forsyth (Eds.), Handbook of social and clinical psychology, Elmsford, NY:Pergamon, 1991.

World Health Organization (WHO). The ICD-10 classification of mental and behavioral disorders. Diagnostic criteria for research. 1993, Geneva. 